\title{
Anterograde Trafficking of CXCR4 and CCR2 Receptors in a Prostate Cancer Cell Line
}

\author{
Kelsie Gillies ${ }^{\mathrm{a}}$ Jaime Wertman $\quad$ Nicholle Charette Denis J. Dupré \\ Department of Pharmacology, Faculty of Medicine, Dalhousie University, Halifax, NS, Canada;

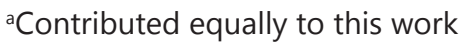

\section{Key Words}

G protein coupled receptor (GPCR) - Prostate cancer metastasis - Rab GTPase • CXCR4 chemokine receptor $\bullet$ CCR2 chemokine receptor $\bullet$ Focal adhesion kinase

\begin{abstract}
Background: Most prostate cancer-related deaths result from metastasis. CXCR4 and CCR2 are known to govern cellular processes resulting in cell migration, proliferation and survival. These receptors are expressed at low levels on normal prostate cells and are highly expressed on malignant and metastatic prostate cancer cells. Signaling of these receptors is relatively well understood, but processes governing their expression at the cell membrane are not. PC3 prostate cancer cells were used to demonstrate the importance of various Rab GTPases on cell surface expression and signaling of CXCR4 and CCR2, along with the CXCR4/CCR2 heterodimer. Methods: PC3 prostate cancer cells were transfected with select Rab GTPase wild-type and dominant negative constructs. Effects of each Rab GTPase on endogenous cell surface expression of the individual receptors, along with the overexpressed CXCR4/ CCR2 heterodimer, were determined by biotin-streptavidin cell surface assays. These results were corroborated by assessing signal transduction, measured by focal adhesion kinase (FAK) activation. Conclusion: Rab GTPases required for cell surface expression and signal transduction of CXCR4 or CCR2 differ from those required for the CXCR4/CCR2 heterodimer. Determining trafficking regulators of two key receptors involved in the metastatic transition may identify new targets to restrict expression of chemokine receptors employed during metastasis.
\end{abstract}


Gillies/Wertman/Charette/Dupré: CXCR4 and CCR2 Trafficking in PC3 Cells

\section{Introduction}

Globally, prostate cancer is second only to lung cancer as the most frequently diagnosed cancer in males. In economically developed countries, it is the most frequently diagnosed cancer in males [1]. Prostate cancer incidence rates vary by at least 25 -fold worldwide largely due to the adoption of prostate-specific antigen testing for malignant growths of the prostate [1]. Although active surveillance of potential malignancies may enhance clinical outcomes, the metastatic propensity of prostate cancer, especially to the bone, acts to decrease survival rates [2].

Numerous G protein coupled receptors (GPCRs), along with their respective ligands, have been identified as integral components that are associated with malignant transformation of prostate cancer cells [3-9]. Within the GPCR superfamily, chemokine receptors have proven to be particularly interesting to study in relation to prostate cancer development and progression. Since chemokines are produced not only by cancer cells, but also by a myriad of other cell types associated with cancer [10], there is an extensive web of interactions between chemokines and their receptors. The importance of these interactions is accentuated by the fact that chemokines and their respective receptors have been implicated in numerous processes that favor cancer progression, including, but not limited to: growth, invasion, metastasis and angiogenesis [11].

C-X-C chemokine receptor type 4 (CXCR4) is an example of a GPCR that has been implicated in the metastasis of cancer. Enhanced CXCR4 expression is present in localized and metastatic prostate cancer [12] and is generally associated with both lower survival rates [13] and more aggressive phenotypes [14]. Signaling through CXCR4 upon activation by stromal derived factor 1- $\alpha$ (SDF-1 $\alpha$; CXCL12) has been shown to enhance both perineural invasiveness of prostate cancer cells [15] and prostate cancer cell adhesion to bone marrow endothelial cells [16]. SDF-1 $\alpha$ is highly expressed in common sites of prostate cancer metastasis such as the bone, kidney and liver, in comparison with relatively low expression at rare sites of prostate cancer including the tongue and eye [17]. Furthermore, administration of a CXCR4 neutralizing antibody or blocking peptide decreased intraosseous tumor burden following intratibial tumor cell injections and also reduced bone metastasis following intracardiac injection of prostate cancer cells [17]. Similarly, AMD3100 (a CXCR4 antagonist) inhibited prostate cancer cell invasiveness [15].

Another chemokine GPCR, C-C chemokine receptor type 2 (CCR2), is also expressed prominently on malignant prostate cancer lesions and expression increases with pathologic stage [18]. The main ligand for CCR2, monocyte chemotactic protein-1 (MCP-1; CCL2), has been shown to be a chemoattractant for prostate cancer cells, in addition to its ability to enhance cell division and invasion of these cancer cells [19]. MCP-1 has not only demonstrated angiogenic potential [20], but it is also believed to promote prostate cancer-induced osteoclastogenesis [21]. A murine xenograft model utilizing a prostate cancer cell line demonstrated decreased tumor burden and macrophage infiltration upon administration of CCL2 neutralizing antibodies [22], suggesting an important role for this chemokine in prostate cancer progression.

Physiologically, GPCRs have been observed as homo- or heterodimers, along with higher-order oligomers [23]. Homodimerization has been demonstrated for both CXCR4 [24-26] and CCR2 [25, 27, 28]. It has also been reported that CXCR4 and CCR2 can form heterodimers $[25,29]$. Further study of the CXCR4/CCR2 heterodimer found that AMD3100 impaired interaction of MCP-1 with its receptor in the CXCR4/CCR2 receptor complex [29]. Analogously, the CCR2 antagonist TAK779 was found to decrease SDF-1 $\alpha$ interaction with CXCR4 [29]. Functional consequences relating to chemotactic response and calcium flux were affected accordingly as a result of cross-inhibition between partners of the CXCR4/ CCR2 heterodimer [29].

While the signal termination processes of GPCRs are relatively well known, such as desensitization and endocytosis, processes governing assembly of receptor signaling complexes and their intracellular trafficking to the plasma membrane is generally not well 
Gillies/Wertman/Charette/Dupré: CXCR4 and CCR2 Trafficking in PC3 Cells

characterized. The expression of CCR2 and CXCR4 at the plasma membrane is of interest to cancer researchers because their respective ligands are able to cause cancer cell migration, and possibly increase the likelihood and severity of metastasis. Therefore, the pathways governing their cell surface expression as both individual receptors and heterodimers are significant processes that warrant investigation to help understand cancer progression and subsequent metastasis. Characterization of the trafficking pathways used by chemokine receptors CXCR4 and CCR2 for their expression at the plasma membrane could help identify targets that could be used to restrict their expression at the cell surface. Individual Rab GTPases have been used to control transport steps associated with receptor transport from the endoplasmic reticulum (ER) to plasma membrane [30-32]. Specifically, effects of the dominant-negative Rab1, 2, 6, 8 and 11 isoforms were investigated because these Rab GTPases are known to play a role in the transport of GPCRs from the ER to the plasma membrane [30-32]. We demonstrate how different Rab GTPases regulate anterograde trafficking of endogenous CXCR4 and CCR2 receptors and overexpressed CXCR4/CCR2 heterodimers in a prostate cancer cell line.

Although there are a wide variety of signal transduction cascades initiated by activation of GPCRs [33], we chose to concentrate on how the activation of focal adhesion kinase (FAK), a non-receptor tyrosine kinase that has been shown as a key regulator of cell growth, survival and migration - key processes often altered in cancer progression [34], is affected by anterograde trafficking of chemokine receptors.

\section{Materials and Methods}

\section{Reagents}

PC3 cells were obtained from ATCC (Manassas, VA). Fetal bovine serum and Lipofectamine 2000 were purchased from Invitrogen (Etobicoke, ON, Canada). Dulbecco's Modified Eagle's Medium (DMEM) High Glucose, monoclonal anti-FLAG antibody, protein A-Sepharose and all chemicals were from Sigma-Aldrich (Oakville, ON, Canada). Laemmli sample buffer was from BioRad (Mississauga, ON, Canada) and BioTrace ${ }^{\mathrm{TM}}$ nitrocellulose transfer membrane was from Pall Corporation (Port Washington, NY, USA). Supersignal West Femto Maximum Sensitivity Substrate, EZ-link Sulfo-NHS-LC-Biotin and Streptavidin Agarose Resin were from Pierce Biotechnology (Rockford, IL, USA). SDF-1 $\alpha$, MCP-1 and monoclonal anti- $\beta$ actin were from Abcam (Toronto, ON, Canada). Polyclonal anti-GFP, monoclonal anti-c-myc, monoclonal anti-CCR2, polyclonal anti-fusin (C-20; anti-CXCR4), monoclonal anti-pFAK and horseradish peroxidase (HRP)conjugated secondary antibodies were from Santa Cruz Biotechnology (Santa Cruz, CA, USA). Polyclonal anti-FAK was from Biovision (Milpitas, CA, USA).

\section{Constructs}

CCR2 and CXCR4 receptors were purchased from the Missouri University of Science and Technology cDNA resource center and subcloned into a pcDNA3.1 vector containing the N- (venus1) or C-terminal (venus2) fragment of venus yellow protein as described in [35]. Wild-type and dominant negative Rab GTPases were constructed as detailed in [32].

\section{Cell culture and transfection}

The PC3 cell line was maintained in DMEM High Glucose supplemented with $10 \%$ fetal bovine serum. Cells were plated in 6-well plates and subsequently transfected with cDNA using Lipofectamine 2000 according to manufacturer's instructions. Experiments were normally conducted $48 \mathrm{~h}$ post-transfection, following $24 \mathrm{~h}$ of serum deprivation.

\section{Fluorescence Microscopy}

Twenty four hours following transfection of PC3 cells with CXCR4 V1 and CCR5 V2, cells were harvested and seeded onto laminin-coated cover slips and incubated at $37^{\circ} \mathrm{C}$ for four $4 \mathrm{~h}$. PC3 cells were then fixed using 3\% (w/v) paraformaldehyde in PBS, pH 7.4 for $20 \mathrm{~min}$ at room temperature. Cover slips were then rinsed with PBS and mounted onto glass slides with $0.4 \%$ 1,4-diazabicyclo\{2.2.2\}octane/glycerol medium 
Gillies/Wertman/Charette/Dupré: CXCR4 and CCR2 Trafficking in PC3 Cells

and sealed using nail polish. Microscopy was done using an Olympus IX81 microscope equipped with a Photometrics coolSNAP HQ2 camera and an excite series 120Q light source. The venus protein (YFP) was excited at $488 \mathrm{~nm}$ and images were acquired at $525 \mathrm{~nm}$.

SDS-PAGE gel electrophoresis and western blotting

PC3 cells were treated with SDF- $1 \alpha$ or MCP- 1 for $15 \mathrm{~min}$ at $37^{\circ} \mathrm{C}$, or remained unstimulated. Cells were lysed in radioimmume precipitation assay (RIPA) buffer (50 mM Tris (pH 7.4), $100 \mathrm{mM} \mathrm{MgCl} \mathrm{z}_{2}, 150 \mathrm{mM} \mathrm{NaCl}$, 0.5\% sodium deoxycholate, $1 \%$ Nonidet P-40, 0.1\% SDS, Roche COMPLETE protease inhibitor tablet) and placed in a dry bath at $65^{\circ} \mathrm{C}$ for $5 \mathrm{~min}$ prior to being resolved by SDS-PAGE and transferred to nitrocellulose membranes. Immunoblots were probed with primary antibodies overnight and then HRP-conjugated secondary antibodies. Immunoblots were visualized with Western Lightning Plus-ECL Chemiluminescence Substrate. Image 4.3 software (NIH) was used to calculate band densities of the immunoblots.

Biotin-streptavidin cell surface assay

PC3 cells were transfected with a venus-tagged receptor pair, as well as a Rab construct, and harvested after 48 h. Cells were washed with PBS prior to incubation with $0.9 \mathrm{mM}$ EZ-link Sulfo-NHS-LC-Biotin for 30 min. Samples were washed with $100 \mathrm{mM}$ Glycine-PBS and lysed with RIPA buffer. Supernatants were incubated overnight with Streptavidin Agarose Resin. Three washes with RIPA buffer were completed before beads were incubated in SDS-PAGE sample buffer with 2.8 M DTT for $1 \mathrm{~h}$ at room temperature to elute bound proteins. Immunoblots were probed with anti-GFP antibody, anti-CCR2 or anti-CXCR4, as well as a horseradish-peroxidase-conjugated secondary antibody.

\section{Co-immunoprecipitation}

$100 \mu \mathrm{l}$ of RIPA buffer was used to lyse cells and samples were pre-cleared with $10 \mu \mathrm{l}$ of protein-A sepharose beads with BSA and placed to nutate for 30 minutes at $4^{\circ} \mathrm{C}$. Lysates were then clarified by centrifugation at 13000 RPM for 15 minutes at $4^{\circ} \mathrm{C}$. Samples were incubated with anti-CXCR4 antibody for 30 minutes at $4^{\circ} \mathrm{C}$. The subsequent protein-antibody complex was precipitated with $50 \mu$ l of protein-A sepharose beads at $4^{\circ} \mathrm{C}$ to precipitate proteins of interest. Samples were then washed with RIPA and proteins were eluted with $100 \mu \mathrm{l}$ of Laemmli sample buffer containing $\beta$-mercaptoethanol. For cell lysis analysis, $30 \mu \mathrm{l}$ of the supernatant that resulted from centrifugation was added to $30 \mu \mathrm{l}$ of Laemmli sample buffer containing $\beta$-mercaptoethanol. Samples were processed with SDS-PAGE and immunoblots were probed with anti-CCR2 and anti-CXCR4 antibodies.

\section{Results and Discussion}

CXCR4 and CCR2 expression in PC3 cells

Constant improvements have been made not only to the diagnosis, but also to the treatment of prostate cancer [36]. Unfortunately, metastatic prostate cancer is still associated with poor prognosis [36, 37]. PC3 cells have demonstrated higher metastatic potential compared to other cell lines [38]. Previous studies have shown endogenous expression of the chemokine GPCRs CXCR4 [12] and CCR2 [18] in the PC3 cells, suggesting that these receptors may contribute to heightened malignancy of the cancer. Anti-CXCR4 antibodies also significantly impaired the migration and invasive potential of PC3 cells [39]. For these reasons, the PC3 human prostate cancer cell line was chosen for the following experiments. Endogenous levels of CXCR4 and CCR2 expressed endogenously on PC 3 cells were evaluated using western blotting; the results are shown in Figure 1A and IB. It has been demonstrated that these receptors form both homodimers and heterodimers, and that ligands interact differentially with the heterodimer than the individual receptors [25, 29]. As shown by co-immunoprecipitation, endogenous CXCR4 can co-precipitate with endogenous CCR2 in the PC3 cell line (Fig. 1A). Additionally, the same experiment was done in the opposite order; immunoprecipitating CCR2 and blotting against CXCR4 yields the same result (Fig. 1B). Therefore, these two chemokine receptors form a signaling complex with each other at endogenous expression levels in PC3 cells. Signaling resulting from receptor activation of 
A)

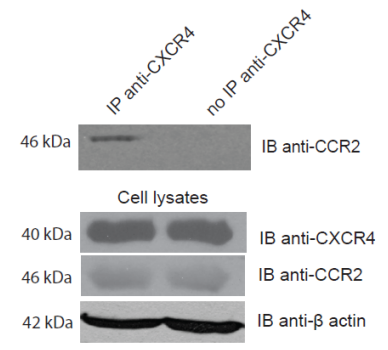

C)

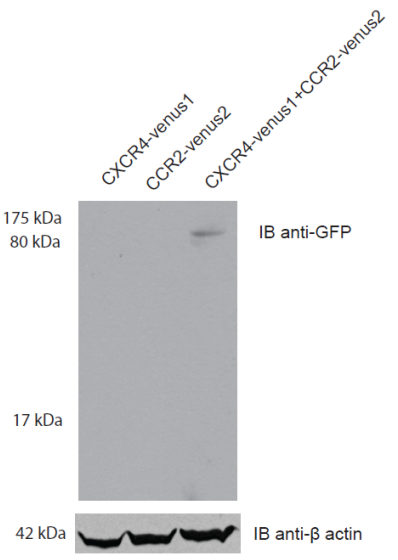

E)

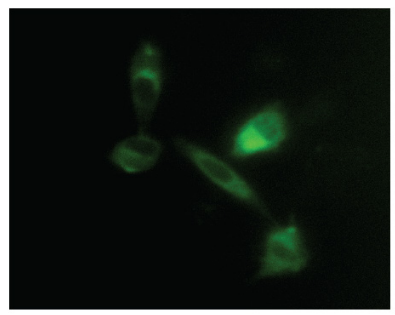

CXCR4-venus1 + CCR2-venus2 in PC3 Cells
B)

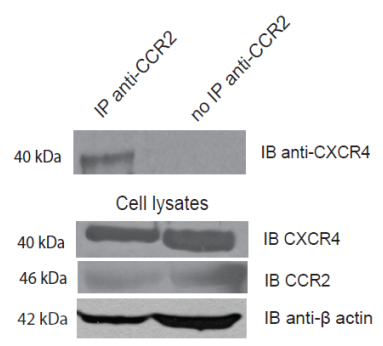

D)
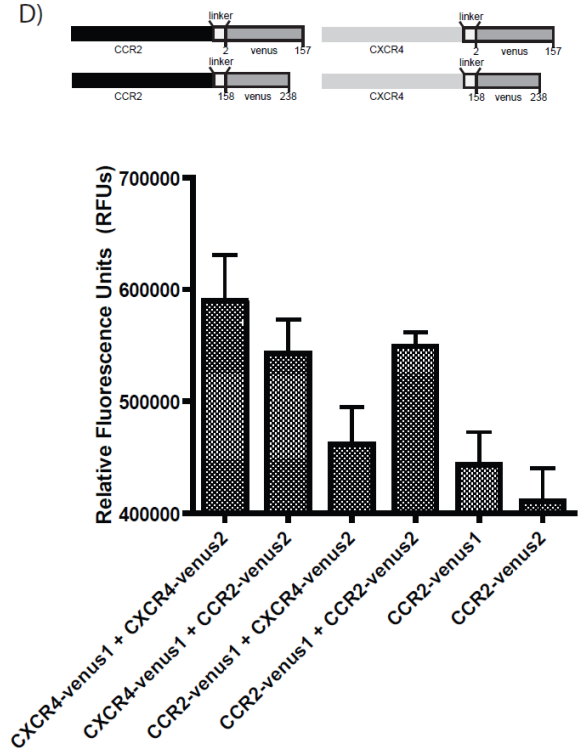

Fig. 1. Expression and interaction of chemokine receptors in PC3 cells. A) Co-immunoprecipitation of the CXCR4/CCR2 complex in PC3 cells. PC3 cells expressing endogenous receptors were lysed and immunoprobed with anti-CXCR4 (1:1000). Samples were processed with SDS-PAGE and immunoblotted with anti-CCR2 (1:1000). Lysates were immunoblotted with anti-CXCR4 (1:1000), anti-CCR2 (1:1000) or anti- $\beta$ actin (1:5000). B) Same experiment as shown in A), but probing against CCR2 and blotting against CXCR4. C) Specificity of GFP antibody to the CXCR4v1/CCR2v2 heterodimer. Cells were transfected with CXCR4v1, CCR2v2 or CXCR4v1/CXCR4v2. $48 \mathrm{~h}$ post transfection, cells were lysed in RIPA buffer and western blot analysis was completed with anti-GFP (1:1000) to indicate the presence of the YFP variant, venus. D) Schematic representation of the constructs used for BiFC of the CXCR4/CCR2 heterodimer. The histogram shows the levels of fluorescence observed by the combination of various pairs of BiFC constructs $(n=3)$. E) Sample of fluorescence microscopy demonstrating the CXCR4v1/CCR2v2 pairing exhibiting fluorescence. Venus (YFP) was excited at $488 \mathrm{~nm}$ and the images were acquired at $525 \mathrm{~nm}$.

the heterodimer complex may differ from that which is expected for the individual receptors, warranting further investigation.

To facilitate the study of this complex, bimolecular fluorescence complementation (BiFC) was used. BiFC allows for direct visualization of protein-protein interactions in living 
Fig. 2. Expression of Rab GTPases, as well as total endogenous CXCR4 and CCR2 receptors, in PC3 cells transfected with WT and dominant negative Rab constructs. $48 \mathrm{~h}$ post transfection, cells were harvested and lysed with RIPA buffer and processed with SDS-PAGE. Membranes were probed with antimyc (1:1000), anti-flag antibodies (1:1000), anti-CXCR4 (1:1000), antiCCR2 (1:1000) antibodies or anti- $\beta$ actin (1:5000).

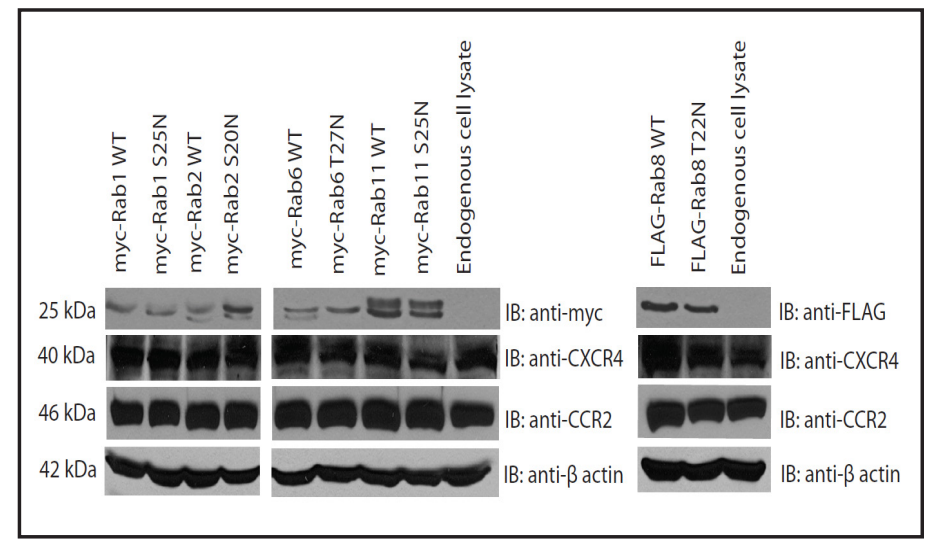

cells through the formation of a fluorescent molecular complex. Two protein fragments, each of which is unable to fluoresce individually, fuse and form a fluorescent protein [40]. Most often, variants of yellow fluorescent protein are used such that two halves are created - an $\mathrm{N}$-terminal and C-terminal fragment [40]. In this study, the Venus variant of yellow fluorescent protein was used and either an N-terminal (Venus1, the first 157 amino acids of Venus) or C-terminal (Venus2, amino acids 158 to 238 of Venus) fragment was coupled to a receptor. When not reconstituted, each singular Venus fragment is unable to emit fluorescence and cannot bind to an anti-GFP antibody. Upon dimerization of receptors coupled to each Venus fragment, Venus1 and Venus2 form a functional Venus that emits fluorescence and can bind to an anti-GFP antibody (Fig. 1C). Figure 1D and 1E show the relative fluorescence levels of various chemokine receptor dimer combinations, and fluorescence microscopy of the CXCR4/CCR2 heterodimer pairing used in the study.

\section{Rab expression}

Anterograde trafficking is a critical step in the regulation of GPCR signaling; the assembly and trafficking of receptor complexes depends on a number of factors, including Rab GTPases. It has been shown that anterograde trafficking pathways of multiple GPCRs can be altered by transfection with dominant negative forms of Rab GTPases in numerous cell lines, including HEK293s [30, 32], Jurkats [41] and cardiac myocytes [31]. Here, we sought to investigate how affecting receptor trafficking with various Rab GTPases could alter plasma membrane expression of endogenous CXCR4 and CCR2, as well as the CXCR4/CCR2 heterodimer. PC3 cells were transfected with myc- or flag-tagged WT or dominant negative Rab GTPases constructs for $48 \mathrm{~h}$, lysed and loaded on SDS-PAGE. Figure 2 shows that expression levels for each Rab GTPase were similar among all constructs when transfected into PC3 cells. Therefore, variations in expression levels of the chemokine receptors at the plasma membrane does not originate from various levels of expression of the GTPases, but rather from the regulation of receptor trafficking by the GTPases. Figure 2 also indicates that total endogenous CXCR4 and CCR2 levels are not changed following transfection with Rab GTPase WT or dominant negative constructs.

\section{CXCR4 trafficking pathway}

In order to study the effects of Rab GTPase dominant negative mutants on the trafficking of endogenous CXCR4 and CCR2 and overexpressed heterodimeric complexes to the plasma membrane, we decided to examine cell surface expression of these receptors. PC3 cells were transfected with various Rab GTPase isoforms (WT or dominant negative). Cell surface expression of CXCR 4 was measured $48 \mathrm{~h}$ post transfection by biotin labeling of cell surface proteins, followed by precipitation of the biotin-labeled membrane proteins with streptavidin agarose beads. Western blot analysis with an anti-CXCR4 antibody demonstrated varying levels of endogenous CXCR4 plasma membrane expression that changed depending on the 


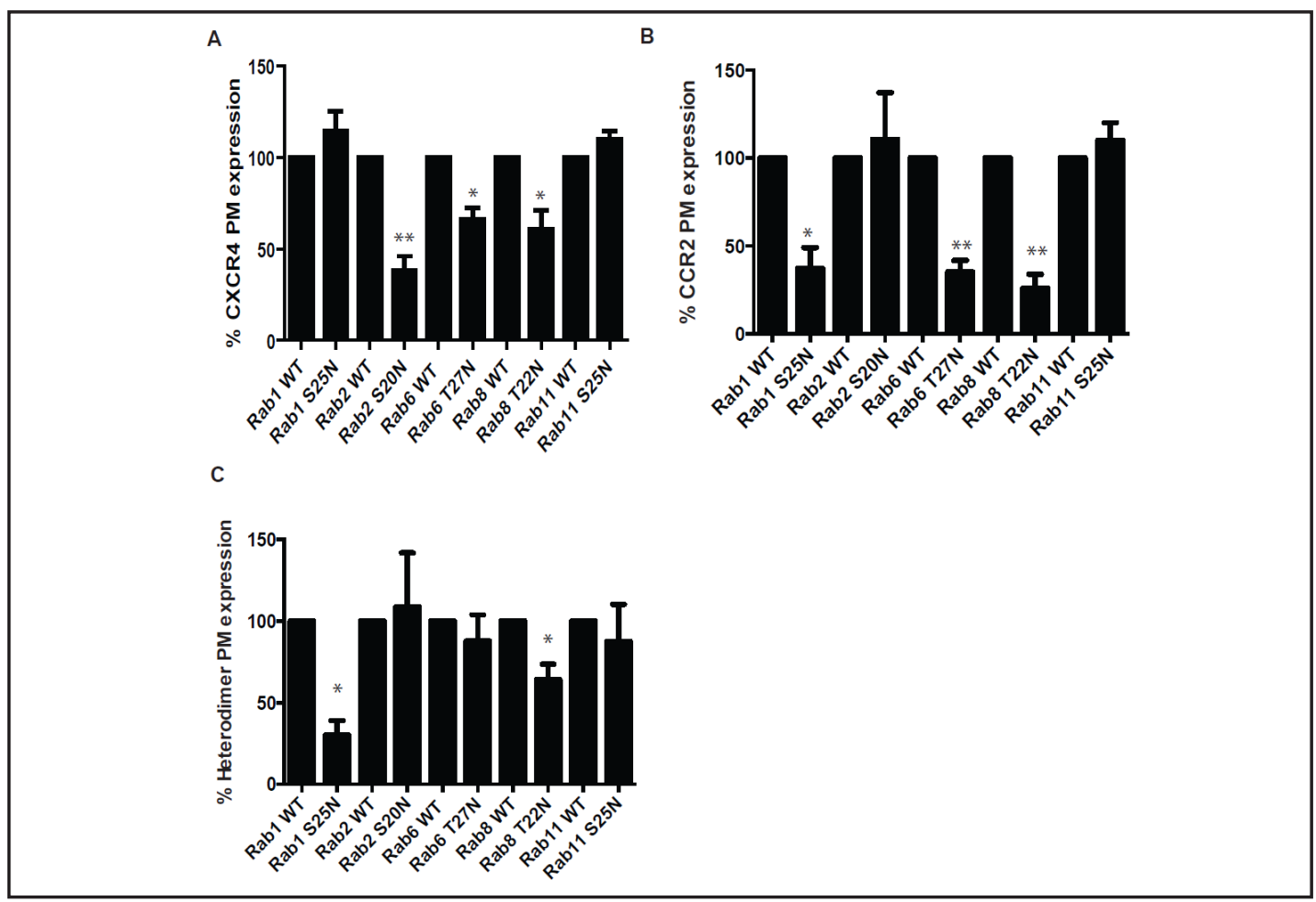

Fig. 3. Effects of dominant-negative Rab GTPase mutants on cell surface expression of CXCR4, CCR2 and the CXCR4V1/CCR2V2 heterodimer in PC3 cells. PC3 cells expressing endogenous receptors were transfected with either Rab GTPase WT or the dominant-negative mutant and subsequent cell surface expression of A) endogenous CXCR4, B) endogenous CCR2 and C) the CXCR4v1/CCR2v2 heterodimer was measured by means of biotin-streptavidin cell surface assays, followed by SDS-PAGE. Immunoblots were probed with anto-CXCR4 (1:1000), CCR2 (1:1000) or anti-GFP (1:1000). Results are expressed as means \pm SEM. * $=$ p $<$ $0.05,{ }^{* *}=\mathrm{p}<0.01,{ }^{* * *}=\mathrm{p}<0.001$ when compared with the Rab WT stimulated samples, using a two-tailed Student's $t$ test.

Rab GTPase isoform expressed by the cells. When compared to their wild-type equivalents, neither Rab1 S25N nor Rab11 S25N affected CXCR4 expression at the plasma membrane (Fig. 3A). However, Rab2 S20N (38.25 $\pm 7.71 \%)$, Rab6 T27N (66.25 $\pm 6.10 \%)$ and Rab8 T22N $(60.75 \pm 10.27 \%)$ decreased CXCR4 expression at the plasma membrane when compared to their wild-type equivalents. These results suggest that the trafficking itinerary of CXCR4 depends on Rab2, Rab6 and Rab8. Recently, a similar study was completed with Jurkat cells [41]. This study demonstrated that endogenous CXCR4 cell surface expression was not affected by Rab1 S25N or Rab11 S25N expression, but was affected by Rab2 S20N, Rab6 T27N, Rab8 T22N co-expression, suggesting that the effect of Rab GTPases on CXCR4 trafficking is not unique to only PC3 cells, and may in fact be conserved among various cell lines.

\section{CCR2 trafficking pathway}

Similar to the study of the CXCR4 trafficking pathway, biotin-streptavidin cell surface assays coupled with western blot analysis revealed differential endogenous CCR2 cell surface expression, depending on expression of the various Rab GTPase isoforms. Neither Rab2 S20N, nor Rab11 S25N influenced CCR2 plasma membrane expression. However, Rab1 S25N (37.33 $\pm 6.74 \%)$, Rab6 T27N (35.33 $\pm 3.76 \%)$, and Rab8 T22N (26.00 $\pm 4.58 \%)$ decreased CCR2 cell surface expression when compared to their wild-type equivalents (Fig. 3B). Whether this pathway is preserved among various cell lines cannot be properly assessed at this point, as this is the first study to examine the effects of these Rab GTPase isoforms on the trafficking itinerary of CCR2. 

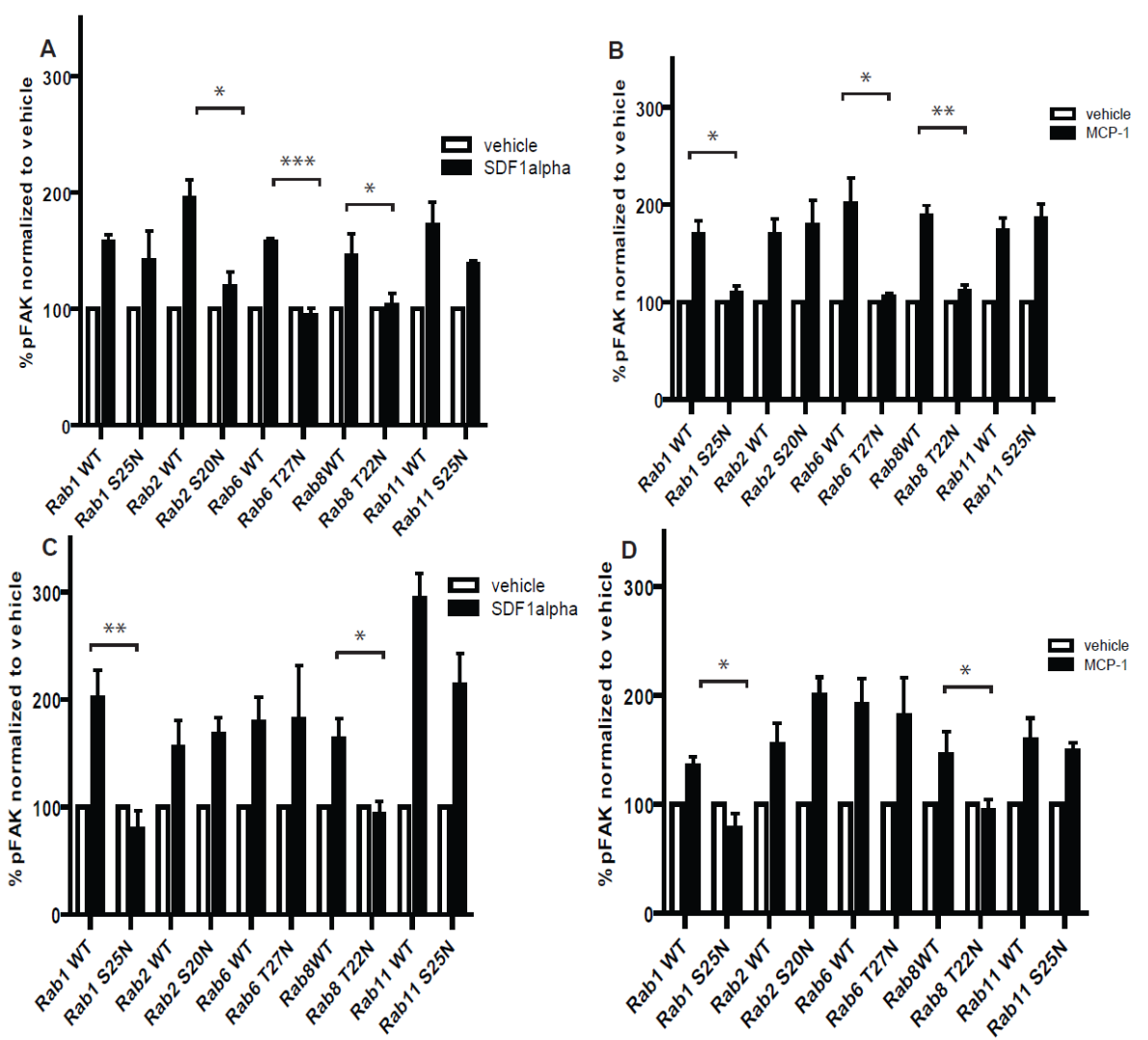

Fig. 4. Effects of dominant-negative Rab GTPase mutants on FAK activation in both the CCR2 and CXCR4 receptors individually and the CXCR4/CCR2 heterodimer in PC3 cells. A) PC3 cells expressing endogenous receptors were transfected with either Rab GTPase WT or the dominant-negative mutant and stimulated with SDF- $1 \alpha$ at $30 \mathrm{ng} / \mathrm{ml}$ for $15 \mathrm{~min}$ at $37^{\circ} \mathrm{C}$ in $5 \% \mathrm{CO}_{2}$, or vehicle. These cells were then lysed and subjected to western blot analysis against p-FAK levels (1:1000). The same membrane was then reprobed for FAK levels (1:1000), as a control. B) Cells transfected the same way as in A) were stimulated with MCP-1 at a concentration of $20 \mathrm{ng} / \mathrm{ml}$ for $15 \mathrm{~min}$ at $37^{\circ} \mathrm{C}$ in $5 \% \mathrm{CO}_{2}$, or vehicle. The cells were then lysed and subjected to western blot analysis. C) PC3 cells were transfected with the CXCR4/CCR2 heterodimer and stimulated with SDF-1 at $30 \mathrm{ng} / \mathrm{ml}$ for $15 \mathrm{~min}$ at $37^{\circ} \mathrm{C}$ in $5 \% \mathrm{CO}_{2}$, or vehicle. Western blot analysis was carried out as in previously described. D) PC3 cells transfected with the CXCR4/CCR2 heterodimer were stimulated with MCP-1 at $20 \mathrm{ng} / \mathrm{ml}$ for $15 \mathrm{~min}$ at $37^{\circ} \mathrm{C}$ in $5 \% \mathrm{CO}_{2}$, or vehicle. The samples were then examined using western blot analysis. Results are expressed as means \pm SEM. ${ }^{*}=p<0.05,{ }^{* *}=p<0.01,{ }^{* * *}=p<0.001$ when compared with the Rab WT stimulated samples, using a two-tailed Student's $t$ test.

\section{CXCR4-CCR2 trafficking pathway}

Biotin-streptavidin cell surface assays coupled with western blot analysis showed that Rab1 S25N and Rab8 T22N significantly (30.33 \pm 8.69\% and $64.18 \pm 9.46 \%$, respectively) decreased plasma membrane expression of the CXCR4V1/CCR2V2 heterodimer (Fig. 3C). In contrast with CXCR4, the heterodimer depends on Rab1 for effective trafficking to the plasma membrane. However, both individual receptors and the heterodimer depend on Rab8 for plasma membrane expression.

\section{FAK Phosphorylation}

FAK is a protein kinase that is involved in the regulation of cell migration downstream from GPCRs, and has therefore been used as a marker for chemokine-induced cell migration 
a)

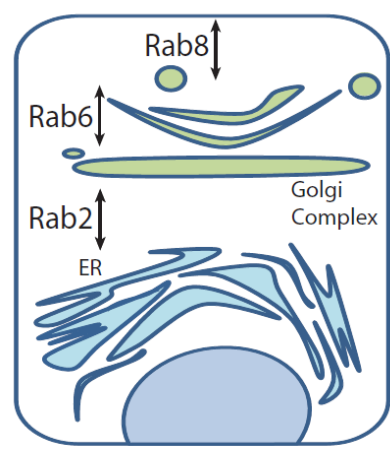

CXCR4

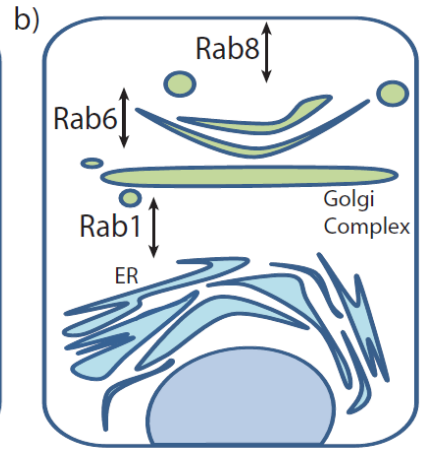

CCR2

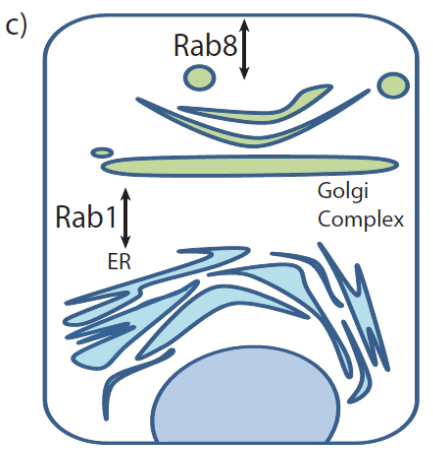

CXCR4/CCR2 heterodimer

Fig. 5. Schematic representation of the Rab GTPases shown to be important for the trafficking of A) CXCR4, B) CCR2 and the C) CXCR4/CCR2 heterodimer in PC3 cells.

[34]. Due to the importance of cell migration in prostate cancer metastasis, we decided to examine activation of the focal adhesion kinase (FAK) in PC3 cells expressing different Rab GTPases isoforms. Plasma membrane is needed for CXCR4 and CCR2 to bind their respective ligands; therefore, studying a signaling pathway that is known to be activated by these receptors would confirm the involvement of Rab GTPases in the regulation of the anterograde trafficking of the receptors, and of the results obtained by cell surface biotinylation.

PC3 cells expressing endogenous receptors were transfected with WT and dominantnegative mutant Rab GTPases and stimulated with the CXCR4 chemokine ligand SDF- $1 \alpha$ at a concentration of $30 \mathrm{ng} / \mathrm{ml}$ for $15 \mathrm{~min}$ at $37^{\circ} \mathrm{C}$ and $5 \% \mathrm{CO}_{2}$ (Fig. 4A). Levels of p-FAK were then quantified with western blotting and compared between non-stimulated and stimulated cells. Results suggest that upon stimulation, there were significant decreases in FAK activation in certain dominant negative treatments compared to the Rab GTPase WT. Cells transfected with Rab2 S20N, Rab6 T27N and Rab8 T22N exhibited statistically significant $(\mathrm{p}<0.05)$ differences in $\mathrm{p}-\mathrm{FAK}$ activation than their WT counterparts, following SDF- $1 \alpha$ treatment ( $\mathrm{p}=0.019,0.0001$ and 0.0258 , respectively). When comparing this to the cell surface assay, it is apparent that cells expressing the dominant negative mutants Rab2 S20N, Rab6 T27N and Rab8 T22N have statistically significant impairment in both CXCR4 trafficking and FAK activation following SDF-1 $\alpha$ stimulation. Since a reduction in cell surface expression of the receptors should lead to an inhibition of downstream signal transduction, here measured as FAK activation, this result corroborates the findings of the trafficking assay.

When PC3 cells were transfected the same way, only stimulated with the CCR2 ligand MCP-1 at a concentration of $20 \mathrm{ng} / \mathrm{ml}$ for $15 \mathrm{~min}$, (Fig. 4A) Rab1 S25N, Rab6 T27N, and Rab8 T22N exhibited statistically significant $(\mathrm{p}<0.05)$ differences in $\mathrm{p}$-FAK activation than the WT Rab GTPases, following MCP treatment $(\mathrm{p}=0.0158,0.0231$, and 0.0035 , respectively; Fig. 4B). Therefore, co-expression of Rab1 S25N, Rab6 T27N and Rab8 T22N inhibited both the expression of CCR2 at the plasma membrane and FAK activation following MCP-1 stimulation.

Next, we measured FAK activation in cells expressing the CXCR4V1/CCR2V2 heterodimer with either MCP-1 or SDF-1 $\alpha$ to determine if there were differences from the endogenous CXCR4 or CCR2 receptors. Results from cells transfected with CXCR4V1/CCR2V2 and the Rab GTPase WTs and dominant negatives demonstrate that upon stimulation with SDF-1 $\alpha$, there are statistically significant $(\mathrm{p}<0.05)$ decreases in p-FAK levels in cells transfected with Rab1 S25N and Rab8 T22N ( $\mathrm{p}=0.0037$ and 0.0078, respectively; Fig. 4C). When PC3 cells expressing the heterodimer and GTPases were stimulated with MCP-1, they showed significant decreases in FAK phosphorylation when Rab1 and Rab8 dominant negative GTPases were expressed ( $p=0.0068$ and 0.0329 , respectively; Fig. 4D). These results correlate 
Gillies/Wertman/Charette/Dupré: CXCR4 and CCR2 Trafficking in PC3 Cells

with what was found in the trafficking assay, as expression of the dominant-negative Rab1 and Rab8 GTPase mutants resulted in impaired expression of the heterodimer at the plasma membrane and a subsequent inhibition of FAK activation following stimulation.

\section{Conclusions and Significance}

Mortality resulting from the metastasis of various cancers, including prostate cancer, justifies close examination of the signal transduction pathways governing this process. Though the roles of the chemokine GPCRs CXCR4 and CCR2 are beginning to be elucidated, the role of the heterodimer is less clear. Similar studies have suggested that heterodimer trafficking itineraries and requirements may be different from each individual receptor, resulting in variable expression at the plasma membrane and subsequent differential signal transduction.

Results relating to cell surface expression of each receptor, along with the CXCR4/CCR2 heterodimer are summarized in Figure 5. We have demonstrated that the CXCR4 receptor requires wild-type Rab2, Rab6 and Rab8 to be expressed normally at the cells surface, and unsurprisingly, requires both Rab2, Rab6 and Rab8 for proper signal transduction, measured as FAK activation. In contrast, the CCR2 receptor depends on Rab1, Rab6 and Rab8 for normal expression at the plasma membrane. Probably as a result of the reduced cell surface expression of the receptors, expression of these Rab GTPases (Rab1, Rab6 and Rab8), decreased the levels of FAK activation.

In contrast with both of the endogenous receptors, this study demonstrates that the CXCR4/CCR2 heterodimer depends on expression of wild-type Rab1 and Rab8 GTPases for proper cell surface expression. When cells expressing the heterodimer and the mutated Rab1 and Rab8 GTPases were stimulated with SDF-1 or MCP-1, there were significant reductions in FAK activation.

Taken together, these results suggest that although the individual receptors have stringent requirements for adequate signaling, the heterodimer depends a different subset of GTPase expression for proper cell surface expression and signaling. The reason for the differences in trafficking requirements between the endogenous CXCR4 and CCR2 receptors vs. heterodimer is as of yet unclear. However, these results are important in terms of developing therapeutics that could be used to prevent or slow prostate cancer metastasis. These studies support the notion that it is important to examine the anterograde trafficking schedules of heterodimeric receptors as an entity distinct from the individual receptor components. Given that the receptors in the present study are known to play a role in some of the signal transduction processes that regulate cell migration, proliferation, and survival, the anterograde trafficking of these receptors is an important aspect of the study of cancer metastasis.

\section{Conflict of Interest}

None declared.

\section{Acknowledgements}

This work is supported by funding from the Canadian Institutes of Health Research (HSH-88136). DJD holds a CIHR New Investigator award. JW is supported by a CIHR Frederick Banting and Best Canada Graduate Scholarship and a Dalhousie Medical Research Foundation De Wolfe Graduate scholarship. KG holds an NSERC PGSD Scholarship and a Killam Predoctoral Scholarship. 
Gillies/Wertman/Charette/Dupré: CXCR4 and CCR2 Trafficking in PC3 Cells

\section{References}

1 Jemal A, Bray F, Cener MM, Feray J, Ward E, Formn D: Global cancer statistics. CA Cancer J Clin 2011;61:6990.

-2 Sturge J, Caley MP, Waxman J: Bone metastasis in prostate cancer: Emerging therapeutic strategies. Nat Rev Clin Oncol 2011;8:357-368.

- 3 Nelson JB, Chan-Track K, Hedica SP, Magnuson SR, Opgenorth TJ, Bova GS, Simons JW: Endothelin-1 production and decreased endothelin B receptor expression in advanced prostate cancer. Cancer Res 1996;56:663-668.

4 Xu L, Stackhouse BG, Florence K, Zhang W, Shanmugam N, Sesterhenn IA, Zou Z, Srikantan, V, Augustus M, Roschke V, Carter K, McLeod, DG, Moul, JW, Soppet D, Srivastava, S: PSGR, a novel prostate-specific gene with homolog to a $\mathrm{G}$ protein-coupled receptor, is overexpressed in prostate cancer. Cancer Res 2000;60:6568-6572.

5 Haese A, Becker C, Noldus J, Gaefen M, Huland E, Huland H, Lilja H: Human glandular kallikrein 2: A potential serum marker for predicting the organ confined versus non-organ confined growth of prostate cancer. J Urol 2000;163:1491-1497.

6 Gohji K, Kitazawa S, Tamada H, Katsuoka Y, Nakajima M: Expression of endothelin receptor a associated with prostate cancer progression. J Urol 2001;165:1033-1036.

7 Porter AT, Ben-Josef E: Humoral mechanisms in prostate cancer: A role for FSH. Urol Oncol 2001;6:131138.

8 Nelson J, Bagnato A, Battistini B, Nisen P: The endothelin axis: Emerging role in cancer. Nat Rev Cancer 2003;3:110-116.

9 Mills GB, Moolenaar WH: The emerging role of lysophosphatidic acid in cancer. Nat Rev Cancer 2003;3:582-591.

10 Lazennec G, Richmond A: Chemokines and chemokine receptors: New insights into cancer-related inflammation. Trends Mol Med 2010;16:133-144.

11 Raman D, Baugher PJ, Thu YM, Richmond A: Role of chemokines in tumor growth. Cancer Lett 2007;256:137-165.

12 Sun Y-, Wang J, Shelburne CE, Lopatin DE, Chinnaiyan AM, Rubin MA, Pienta KJ, Taichman RS: Expression of CXCR4 and CXCL12 (SDF-1) in human prostate cancers (PCa) in vivo. J Cell Biochem 2003;8:462-473.

13 Akashi T, Koizumi K, Tsuneyama K, Saiki I, Takano Y, Fuse H: Chemokine receptor CXCR4 expression and prognosis in patients with metastatic prostate cancer. Cancer Sci 2008;99:539-542.

14 Darash-Yahana M, Pikarsky E, Abramovitch R, Zeira E, Pal B, Karplus R, Avniel S, Kasem S, Galun E, Peled A: Role of high expression levels of CXCR4 in tumor growth, vascularization, and metastasis. FASEB J 2004;18:1240-1242.

15 Zhang S, Qi L, Li M, Zhang D, Xu S, Wang, N, Sun, B: Chemokine CXCL12 and its receptor CXCR4 expression are associated with perineural invasion of prostate cancer. J Exp Clin Cancer 2008;27:62. Doi: 10.1186/1756-9966-27-62.

16 Taichman RS, Cooper C, Keller ET, Pienta KJ, Taichman NS, McCauley LK: Use of the stromal cell-derived factor-1/CXCR4 pathway in prostate cancer metastasis to bone. Cancer Res 2002;62:1832-1837.

-17 Sun Y-, Schneider A, Jung Y, Wang J, Dai J, Wang J, Cook K, Osman, NI, Koh-Paige AJ, Shim H, Pienta KJ, Keller ET, McCauley LK, Taichman RS: Skeletal localization and neuralization of the SDF-1(CXCL12)/CXCR4 axis blocks prostate cancer metastasis and growth in osseous sites in vivo. J Bone Miner Res 2005;20: 318-329.

18 Lu Y, Cai Z, Xiao G, Liu Y, Keller ET, Yao Z, Zhang J: CCR2 expression correlates with prostate cancer progression. J Cell Biochem 2007;101:676-685.

19 19. Lu Y, Cai Z, Galson DL, Xiao G, Liu Y, George DE, Melhem, MF, Yao Z, Zhang J: Monocyte chemotactic protein-1 (MCP-1) acts as a paracrine and autocrine factor for prostate cancer growth and invasion. Prostate 2006;66:1311-1318.

20 Stamatovic SM, Keep RF, Mostarica-Stojkovic M, Andjelkovic AV: CCL2 regulates angiogenesis via activation of ets-1 transcription factor. J Immunol 2006;177:2651-2661.

21 Lu Y, Cai Z, Xiao G, Keller ET, Mizokami A, Yao Z, Roodman, GD, Zhang J: Monocyte chemoattractant protein-1 mediates prostate cancer-induced bone resorption. Cancer Res 2007;67:3646-3653. 
Gillies/Wertman/Charette/Dupré: CXCR4 and CCR2 Trafficking in PC3 Cells

22 Loberg R, Ying C, Craig M, Yan L, Snyder LA, Pienta KJ: CCL2 as an important mediator of prostate cancer growth in vivo through the regulation of macrophage infiltration. Neoplasia 2007;9:556-562.

-23 Milligan G: G protein-coupled receptor dimerization: Function and ligand pharmacology. Mol Pharmacol 2004;66:1-7.

$>24$ Vila-Coro AJ, Rodrígues-Frade JM, Martín de Ana A, Moreno-Ortíz MC, Martínez-A C, Mellado M: The chemokine SDF-1a triggers CXCR4 receptor dimerization and activates the JAK/STAT pathway. FASEB J 1999;13:1699-1710.

25 Percherancier Y, Berchiche YA, Slight I, Volkmer-Engert R, Tamamura H, Fuji, N, Heveker N: Bioluminescence resonance energy transfer reveals ligand-induced conformational changes in CXCR4 homo- and heterodimers. J Biol Chem 2005;280:9895-9903.

26 Wang J, He L, Combs CA, Roderiquez G, Norcross MA: Dimerization of CXCR4 in living malignant cells: Control of cell migration by a synthetic peptide that reduces homologous CXCR4 interactions. Mol Cancer Ther2006;5:2474-2483.

27 Rodrígues-Frade JM, Vila-Coro A, Martín de Ana A, Albar JP, Martínez-A. C, Mellado M: The chemokine monocyte chemoattractant protein-1 induces functional responses through dimerization of its receptor CCR2. Proc Natl Acad Sci USA 1999;96:3628-3633.

28 El-Asmar L, Springael J-, Ballet S, Andrieu EU, Vassart G, Parmentier M: Evidence for negative binding cooperativity within CCR5-CCR2b heterodimers. Mol Pharmacol 2005;67:460-469.

29 Sohy D, Parmentier M, Springael J-Y: Allosteric transinhibition by specific antagonists in CCR2/CXCR4 heterodimers. J Biol Chem 2007;282:30062-30069.

30 Wu G, Zhao G, He Y: Distinct pathways for the trafficking of angiotensin II and adrenergic receptors from the endoplasmic reticulum to the cell surface: Rab1-independent transport of a $G$ protein-coupled receptor. J Biol Chem 2003;278:47062-47069.

31 Filipeanu CM, Zhou F, Claycomb WC, Wu G: Regulation of the cell surface expression and function of angiotensin II type 1 receptor by Rab1- mediated endoplasmic reticulum-to-golgi transport in cardiac myocytes. J Biol Chem 2004;279:41077-41084.

-32 Dupré DJ, Robitaille M, Ethier N, Villeneuve LR, Mamarbachi AM, Hébert TE: Seven transmembrane receptor core signaling complexes are assembled prior to plasma membrane trafficking. J Biol Chem 2006;281:34561-34573.

33 Lappano R, Maggiolini M: G protein-coupled receptors: Novel targets for drug discovery in cancer. Nature Rev Drug Discov 2011;10:47-60.

34 Golubovskaya VM, Kweh FA, Cance WG: Focal adhesion kinase and cancer. Histol Histopathol 2009;24:503510.

-35 Hammad MM, Kuang Y-, Yan R, Allen H, Dupré DJ: Na ${ }^{+} / \mathrm{H}^{+}$exchanger regulatory factor-1 is involved in chemokine receptor homodimer CCR5 internalization and signal transduction but does not affect CXCR4homodimer or CXCR4-CCR5 heterodimer. J Biol Chem 2010;285:34653-34664.

-36 Brower V: Researchers tackle metastasis, cancer's last frontier. J Natl Cancer Inst 2007;99:109-111.

-37 Budendorf L, Schopfer A, Wagner U, Sauter G, Moch H, Willi N, Gasser TC, Mihatsch MJ: Metastatic patterns of prostate cancer: An autopsy study of 1,589 patients. Hum Pathol 2000;31:578-583.

-38 Pulukuri SM, Gondi CS, Lakka SS, Julta A, Estes N, Gujrati M, Rao JS: RNA interference-directed knockdown of urokinase plasminogen activator and urokinase plasminogen activator receptor inhibits prostate cancer cell invasion, survival and tumorigenicity in vivo. J Biol Chem 2005;28:36539-36540.

39 Singh S, Singh UP, Grizzle WE, Lillard JW Jr: CXCL12-CXCR4 interactions modulate prostate cancer cell migration, metalloproteinase expression and invasion. Lab Invest 2004;84:1666-1676.

$>40$ Kerppola TK: Bimolecular fluorescence complementation: Visualization of molecular interactions in living cells. Methods Cell Biol 2008;85:431-470.

41 Charette N, Holland P, Frazer J, Allen H, Dupré DJ: Dependence on different Rab GTPases for the trafficking of CXCR4 and CCR5 homo or heterodimers between the endoplasmic reticulum and plasma membrane in Jurkat cells. Cell Signal 2011;23:1738-1749. 